\title{
Die Glottis.
}

\author{
Von \\ Dr. P. J. Mink. (Deventer.)
}

(Mit 10 Textfiguren.)

Gewöhnlich wird der Teil des Respirationstraktus, der sich oberhalb der Glottis befindet, mit dem Namen "obere Luftwege“ angedeutet. In ihrer Gesamtheit stellen sie einen Raum dar, der während der Atmung immerfort mit Luft angefüllt ist. Dieser Luftraum ist zwischen der Aussenluft und dem eigentlichen subglottischen Atmungsapparate eingeschaltet. Er kann als ein Ansatzstück der Trachea betrachtet werden, wodureh diese indirekt mit der Atmosphäre in Verbindung steht.

Bis jetzt galt dieser obere Luftraum nur als Vorportal für die eintretende und als Resonanzkasten für die austretende tönende Luft. Manometrische Untersuchungen ${ }^{1}$ ) haben aber gezeigt, dass die respiratorischen Drucksehwankungen in hohem Maasse durch diesen Raum beeinflusst werden. Damit musste die Frage aufkommen, ob er für die Atmung selbst und demzufolge auch für den Stoffwechsel von Bedeutung sei.

Klinisch war diese Frage schon in bejahendem Sinne beantwortet, da man die Nachteile der Mundatmung wohl erkannt hatte. Hierbei wird nämlich der obere Luftraum geöffnet und in direkte Verbindung mit der Aussenluft gesetzt. Dadurch werden die respiratorischen Druckschwankungen in ihm nahezu aufgehoben, so dass man von einer freien Kommunikation der Trachea mit der Aussenluft reden kann. Es zeigte sich nun, dass diese Ausschaltung des oberen Luftraumes als Ansatzstück fast regelmüssig die Gesundheit beeinträchtigte.

Sobald man den Druckgrössen einen bedeutenderen Einfluss auf den Atmungsprozess zukennt, kommen verschiedene Vor-

1) Schutter, Annal. d. malad. de l'or. etc. de Gougenheim. 1892. 
gänge in ein anderes Licht. So wurde es uns möglich in der Beweglichkeit der Naseneingänge eine Vorrichtung von hohem physiologischen Werte zu erkennen ${ }^{1}$ ).

Zugleich wurde damit unsere Aufmerksamkeit auf die Glottis hingezogen. Denn Nasenventil und Glottis stehen in etwa gleicher Beziehung zum oberen Luftraume. Sie beherrschen nämlich die beiden Zugänge zu diesem Raume, aber nicht zu gleicher Zeit. Während die Nasenventile bei der Inspiration ais Pförtner fungieren, fällt der Glottis die nämliche Rolle bei der Exspiration zu. Wie von selbst stellt man sich die Frage, ob auch diese letzte von gleich grosser Bedeutung für die Respiration sei wie die Funktion der erstgenannten.

Die beiden Zugänge zum oberen Luftraume liegen in Flächen, die wagerecht aufeinanderstehen. Die nasale, die für uns durch die inneren Nasenlöcher repräsentiert wird, ist frontal gestellt, indem die tracheale nahezu horizontal zur Körperachse steht. Das steht im Einklang mit den Schenkeln, woraus man sich den Luftraum zusammengesetzt denken kann. Denn der Pharynx, wozu die Glottis den Zugang bildet, liegt in der Achsenrichtung der Trachea, während der nasale Luftweg rechtwinklig zu dieser Achse verläuft. - Am besten kann man sich diese Verhältnisse durch nebenstehendes Schema vergegenwärtigen. Hierbei haben wir den Pharynx als überall gleich weit und die Conchae als einfache Verengung des Luftweges vorgestellt. (Fig. 1.)

Wie gesagt, drängt das Verhalten zum oberen Luftraume uns dazu, die beiden Klappenapparate an seinen Enden vom gleichen Gesichtspunkte aus zu betrachten. Nachdem wir das Nasenventil beschrieben haben, ist demnach der erste Schritt die Glottis näher ins Auge zu fassen.

Bei der Vergleichung der beiden Apparate fällt sofort einigeÄhnlichkeit auf. Das Septum nasi wirkt zwar etwas verwirrend, aber man kann es sich wegdenken. Umgekehrt würde man die Mittellinie der Glottis als ein immaterielles Septum betrachten können. In beiden Fällen wird es uns deutlich, dass wir mit Doppelapparaten zu schaffen haben. Klappenpaare, die sich beim Funktionieren einer zwischenliegenden Mitte nähern, bilden das Essentielle beider Vorrichtungen. Beide sind also eingericbtet auf die Einengung

1) P. J. Mink, Das Spiel der Nasenfiügel. Pflüger's Arch. Bd. 120. 
der Öffnungen, die sie beherrschen. Beide verfügen aber zugleich über ein System, das der Erweiterung anstrebt. In erster Linie stehen wir also vor der Frage, welche von beiden Funktionen als die primäre zu gelten hat.

Mit Bezug auf die Nasenventile haben wir uns dahin ausgesprochen (siebe „Spiel der Nasenflügel"), dass ihr Zweck auf die inspiratorische Verengung gerichtet sei. Wir schrieben dieser einen grossen physiologischen Nutzen $\mathrm{zu}$, da wir den Wert einer Inspiration nach ihrem Andauern und dem zur Geltung kommenden negativen Drucke bemessen. Die Erweiterung kam an die zweite

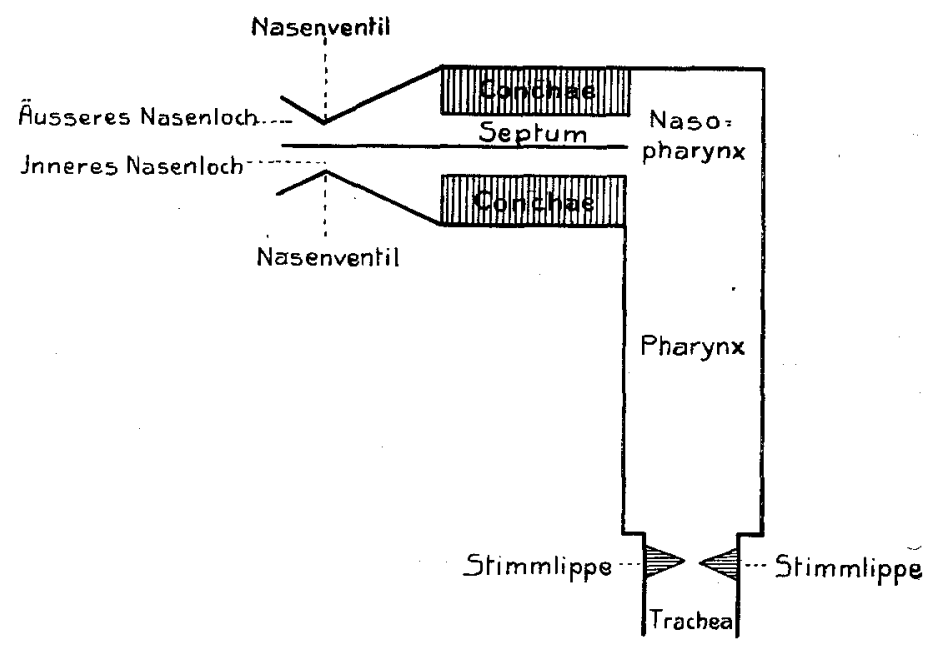

Fig. 1.

Stelle, da sie nur zur Aufgabe hatte, den äussersten Konsequenzen der Verengung vorzubeugen. Wie es in dieser Beziehung mit der Glottis steht, wird uns später klar werden.

Wenn wir ein Nasenventil und eine Stimmlippe mit den von ihnen beherrschten Öffnungen nebeneinander betrachten, so bemerken wir auch eine merkwürdige Ähnlichkeit in der Form. Wir benützen hierzu einen Frontalschnitt durch das innere Nasenloch, wie er schon in "Das Spiel der Nasenflügel " angeführt wird, und einen halbierten Horizontalschnitt durch die Glottis. Denkt man sich die Fasern hinter dem Giessbeckenknorpel, die wir mit $a$ angegeben haben, fort, so fällt die Übereinstimmung noch mehr auf. Das sieht einer Licentia poëtica ähnlich, einfach eine feste Masse wegzudenken. Wenn man 
aber darauf achtet, dass diese Masse gleichwie der angrenzende Teil der Stimmritze zum Bewegungsgebiet des Aryknorpels gehört, so ist die genommene Freiheit nicht so sehr gross. (Fig. 2.)

Man fühlt sich wie herausgefordert, eine Parallele zu ziehen zwischen der Membran in $I$ und dem Giessbeckenknorpel in $I I$. Dieser Vergleich gilt vornehmlich der Funktion. Beide Gebilde machen es nämlich möglich, dass der lange Schenkel der Klappe zur Mittellinie heranrückt. Da aber in $I$ dessen langer Schenkel, nämlich die Ala septi, knorplich ist, musste der Teil, der ihm seine Bewegung gestattet, faserig sein. Und da er in II als Stimmband
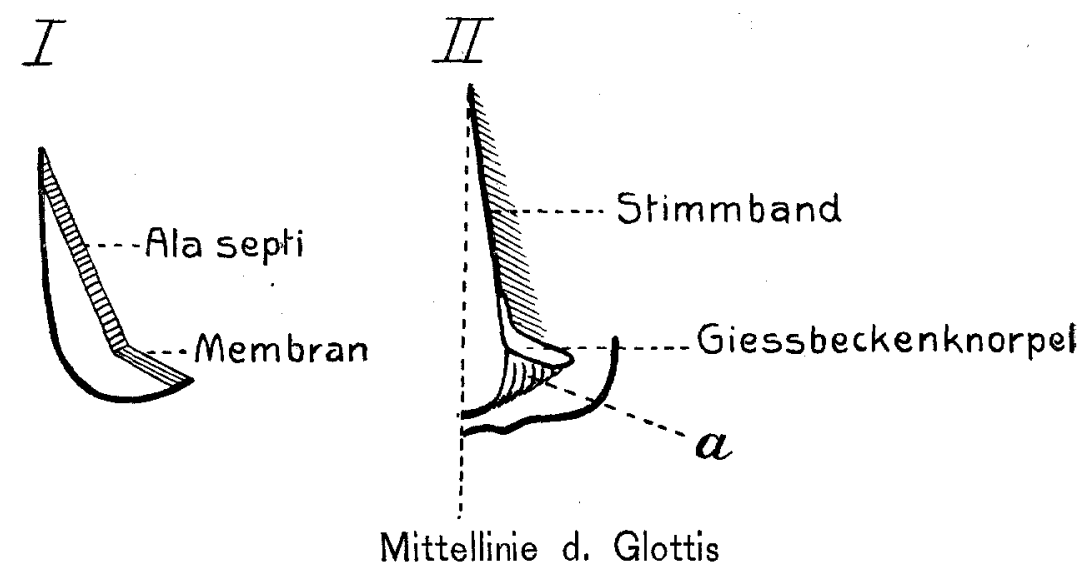

Fig. 2.

faserig ist, so musste der kurze Schenkel knorplich sein. Denn das Festere muss da sein um die Bewegung zu geleiten, und ebenso das Weichere, um sie zu gestatten.

Mit Bezug auf das Nasenventil wird der Satz, dass der Knorpel die Bewegung leitet, wohl nicht auf Widerspruch stossen. Bei der Glottis aber könnte der Umstand, dass das Stimmband einen Muskel in sich birgt, eine andere Auffassung hervorrufen. Das ist auch wirklich geschehen, und die herrschende Kehlkopfphysiologie sagt uns, dass die Spannung der Stimmbänder durch ihre eigene Kontraktion hervorgebracht wird (vgl. Ewald in Heymann's Hdb. Bd. 1 (1) S. 172). Zu ckerkand l sagt wörtlich im nämlichen Buche S. 194: „Dass der Musculus vocalis das Stimmband $\mathrm{zu}$ spannen berufen ist, beweist schon seine Lage in einer Falte, deren Tätigkeit nicht auf Entspannung gerichtet sein kann, ferner der Umstand, dass er ja auf 
die schon vorher von seiten der Cricothyreoidei aus gedehnte Stimmmembran einzuwirken berufen ist." Wir lassen diese Beweisführung dahingestellt sein, konstatieren aber, dass $\mathrm{Zuckerk}$ a nd l eine vorangehende Dehnung gelten lassen will. Wir sind der gleichen Meinung, stellen aber die Frage, ob es wirklich der Musculus cricothyreoideus ist, der diese Dehnung bewirkt.

Das Dehnen setzt ein Auseinanderrücken der Ansatzpunkte voraus. Diese sind einerseits durch den Schildknorpel, andererseits durch die Aryknorpel gegeben. Die Anheftung an der Cartilago thyreoidea muss als fixer Punkt betrachtet werden, denn, wie Ewald (Heymann's Hdb. Bd. 1 [1] S. 196) sagt, ist es sicherlich richtig, dass sich eigentlich nur der Ringknorpel am Schildknorpel bewegt und nicht umgekehrt (Jelénffy, Schech). Der Schildknorpel ist durch die an ihm befestigten Halsmuskeln fixiert und der Ringknorpel dagegen sehr leicht beweglich. Am freigelegten Kehlkopf des Hundes kann man sich von diesen Verhältnissen aufs deutlichste überzeugen, besonders wenn man in die beiden Knorpel lange Nadeln eingestossen hat.

Es bleibt also nur der Giessbeckenknorpel um das Auseinanderrücken der Ansatzpunkte und damit die Dehuung der Stimmembran zu erwirken. Am deutlichsten muss sich der Vorgang an der Chorda vocalis, die eigentlich nur die verdickte Randpartie der Membran darstellt, abspielen. Wir fassen darum diese speziell ins Auge.

Der vordere fixe Ansatzpunkt der Chorda findet sich ziemlich genau am Halbierungspunkte der Höhe des Winkels der Cartilago thyreoidea, dicht neben dem Bande der entgegengesetzten Seite. Der hintere Ansatzpunkt am Processus vocalis des Aryknorpels muss in sagittaler Richtung nach hinten ausweichen, um das Ligament anzuspannen.

Man hat sich die Frage gestellt, durch welche verschiedenartige Bewegungen diese Spannung bewirkt werden kann, und dieses am ausgeschnittenen Kehlkopf ausprobiert. Aber diese Fragestellung ist unrichtig. Man hat nur zu rechnen mit den Bewegungen die wirklich beim Lebenden vorkommen. Fraglich ist nur, welche von diesen die Anspannung hervorruft.

Da kommen in erster Linie die Atembewegungen in Betracht. Laryngoskopisch kann man wahrnehmen, dass eine Exspiration manchmal eine Anspannung hervorruft. Man kann zwar Semon beipflichten, wo er sagt, dass bei der ruhigen Respiration in $80 \%$ 
der Fälle keine Bewegung der Stimmbänder zu sehen ist. Aber bei etwaiger verstärkter Atmung sieht man die Anspannung doch jedesmal deutlich hervortreten. Hier haben wir also einen Konnex vor uns, der in Wirklichkeit besteht und also als Ausgangspunkt für unser Raisonnement dienen kann. Die Frage ist, wie wir uns diesen Zusammenhang zu denken haben.

Es ist bekannt, dass die Trachea sich schon infolge der gewöhnlichen Atembewegungen hebt und senkt. Bei der Inspiration zieht das Zwerehfell die Lunge und somit die Luftröhre abwärts, bei der Exspiration findet die entgegengesetzte Bewegung statt. Bei starken Ausatmungsbewegungen ist schon deutlich das Aufsteigen des ganzen Kehlkopfes zu sehen.

Im Ruhezustande ist der Larynx aber nicht als eine Einheit zu betrachten. Er zeigt eine Zusammensetzung aus zwei Stücken, die sich bis zu einer gewissen Höhe unabhängig von einander bewegen können.

Das untere der beiden Stücke, nämlich der Ringknorpel, ist nur als ein besonders formierter Trachealring zu betrachten. Er ist mit dem sogenannten obersten Trachealringe verbunden auf eine Weise, die sich in nichts unterscheidet von den Verbindungen der Trachealringe untereinander. Nur gestaltet er durch die Ungleichheit seiner Höhe vorn und hinten den Mund der Luftröhre schief, etwa wie das Mundstück einer Flöte. Durch den festen Zusammenhang muss er die Bewegungen der Trachea so gut wie unverändert mitmachen.

Ganz anders steht es um das obere der beiden Stücke, aus dem der Kehlkopf aufgebaut ist, nämlich um den Schildknorpel. Dieser ist gewissermaassen am Zungenbein aufgehängt und, wie schon bemerkt, durch die vorderen Halsmuskeln fixiert. Da seine Verbindung mit dem Ringknorpel durch Bänder und Gelenke eine ziemlich schlaffe ist, so behauptet er der Luftröhre gegenüber einige Selbständigkeit. Die Muskeln, die ihn mit dem Ringe verbinden, kommen in dieser Beziehung nur bei ihrer Kontraktion in Betracht.

Die relative Unabhängigkeit des Schildknorpels muss aufgehoben werden, bevor der Kehllkopf als Ganzes betrachtet werden darf. Wenn also die Luftröhre und damit der Ringknorpel den Ruhezustand verlässt und sich hebt, hat zuerst diese Aufhebung zu erfolgen. Es muss sich mit anderen Worten das untere Stück des Kehlkopfes erst am oberen Stücke verschieben, bevor es dieses mitbewegen kann. 
Gross ist diese Verschiebung freilich nicht. Ewald untersuchte eine sehr magere Person darauf und fand eine Differenz von höchstens $1 \mathrm{~mm}$. Bei anderen Personen konnte er sie überhaupt nicht konstatieren. (Heymann's Handbuch Bd.1 [1], S. 198.) Man hat aber das Recht zu bezweifeln, ob es möglich sei, genau zu messen ohne Blosslegung des Kehlkopfes. Es wäre wünschenswert, die Messungen beim Menschen in vivo unter obenstehender Bedingung zu wiederholen. Schade, dass Tracheotomierten nur ausnahmsweise für diesen $\mathrm{Zweck}$ zu benutzen sind, da man hier meistens pathologische Fälle vor sich hat.

Es genügt aber für unseren Zweck zu wissen, dass das anatomische Verhältnis uns berechtigt, anzunehmen, dass jede Hebung des Ringknorpels mit einer Verschiebung dem Schildknorpel entlang einzusetzen hat. Diese Bewegung wird sich am deutlichsten da zeigen, wo die beiden Knorpel einander unmittelbar berühren. d. h. also im Cricotbyreoidalgelenk. Dieses Gelenk lässt Schlotterbewegungen in der Richtung nach oben, unten, vorn und hinten zu. Nach Harless ${ }^{1}$ ) erfolgt aber lediglich eine Bewegung in Vertikalebenen.

Mit "vertikale Richtung" wird wohl diejenige gemeint sein, die der Trachealachse entspricht. Wir sehen $a b$ von möglichen Abänderungen in der Richtung dieser Achse selbst, die bei der kleinen Verschiebung, die wir im Auge haben, normaliter ausserhalb des Spieles steht.

Der Processus vocalis ist als ein Punkt einer Trachealachse zu betrachten (Fig. 3). Solange der Aryknorpel seine Stellung zum Ringknorpel nicht ändert, hat dieser Processus als ein Teil der Trachealröhre zu gelten, der nach der Mitte hin gerückt ist. Es macht nichts aus, ob er gerade in der Mittelachse der Trachea liegt; es genügt, dass er sich dieser mehr oder weniger nähert.

Das Stimmband verbindet diesen Punkt der Trachealachse mit dem Schildknorpel. Solange diese Verbindung schlaff ist, kann dieser Punkt sich gleich wie die Achse bewegen. Eine gewisse Rigidität des Zusammenhangs mit dem Ringknorpel gestattet ihm, treu dessen Bewegungen mitzumachen, da er hierauf ruht. Sobald das Band aber angespannt ist, ändert sich die Sache. Von höchster Bedeutung ist es also, zu wissen, wie weit sich die freie Bewegung des Processus vocalis erstreckt.

1) Harless, Artikel „Stimme" in Wagner's Handwörterb. d. Physiol. Bd. 4. 
Am besten vergegenwärtigt man sich die Sachlage an untenstehender Zeichnung, der eine Figur aus Luschka's Kehlkopf zugrunde liegt. (Fig. 3.)

Wir haben von $a$, dem vorderen Ansatz des Stimmbandes, aus einen Zirkelbogell beschrieben, der dessen hinteren Ansatz am Processus vocalis $b$ trifft. Die Linie $e c$ stellt die Trachealachse vor, die man sich durch $b$ gezogen denken kann. Es leuchtet ein, dass der Processus vocalis sich vom Zirkelbogen entfernt, wenn er sich in

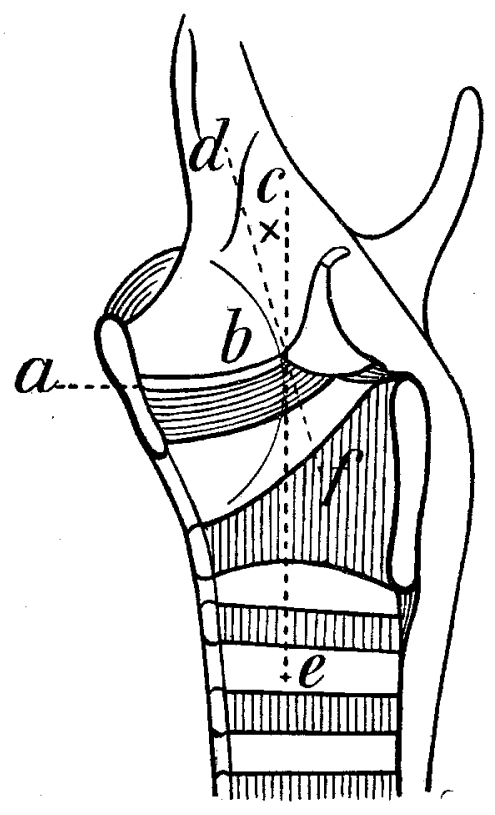

Fig. 3.

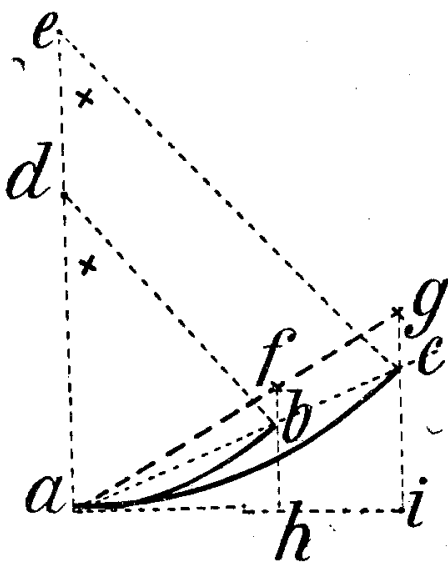

Fig. 4.

der Richtung der Trachealachse nach oben bewegt. Damit wird der Abstand zwischen $a$ und $b$ grösser, so dass das Stimmband angespannt wird, wenn nämlich $a$ fix bleibt. Hieraus geht hervor, dass eine Hebung der Trachea, wie sie bei der Exspiration vorkommt, imstande ist, diese Anspannung zu erwirken.

Die Abstandsvergrösserung zwischen $a$ und $b$ wird bestimmt durch den Winkel, den die Achslinie $c b$ mit dem Zirkelbogen bildet. Ziehen wir durch $b$ eine Tangente $d f$, so ist der Winkel $d b c$ das Maass für diese Vergrösserung.

Wenn man von einer gleichen Erschlaffung des Stimmbandes ausgeht, so ist auch dessen Länge von Bedeutung. Die Schlaftheit 
eines regelmässig gebogenen Bandes kann nämlich bemessen werden nach dem Winkel, welcher der Biegung entspricht. So würde man die Bänder $a b$ und $a c$ (Fig. 4) gleich schlaff nennen dürfen, weil $\angle a d b=\angle a e c$ ist. Wenn $a f$ die Länge des gestreckten Bandes $a b$ vorstellt, so wird $a g$ dem gestreckten Band $a c$ entsprechen müssen. Wenn die Streckung des ersten durch eine Verschiebung von $b$ bis $f$ zustande kommt, so erfordert eine gleichgerichtete Verschiebung von $c$ den Abstand $c g$ um das Band $a c$ zu strecken. Es ist ohne weiteres klar, dass $b f$ und $c g$ sich verhalten wie die Bogenlängen $a b$ und $a c$ und andererseits bestimmt werden durch die Winkel $d b f=e c g=a d b$.

Wir kommen solcherweise zu folgendem Satze: Bei gleicher Stellung und Schlaffheit der Stimmbänder ist die

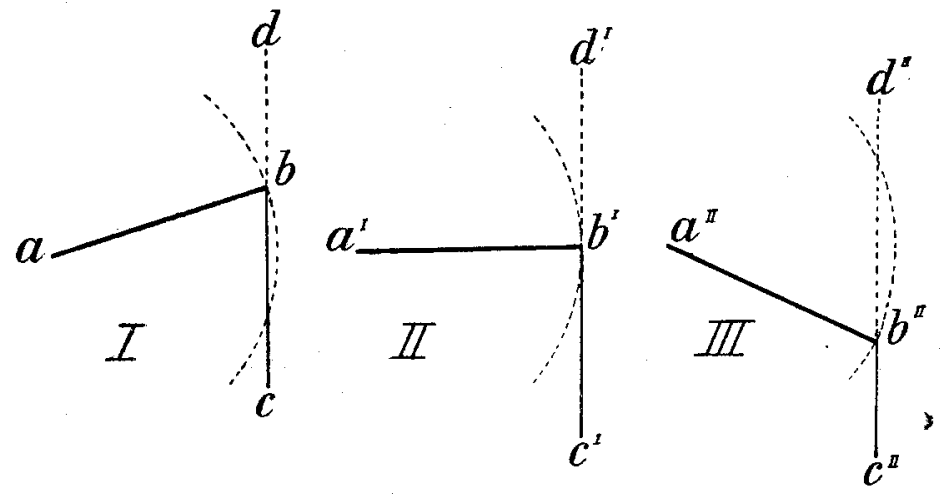

Fig. 5 .

zur Streckung der Bänder erforderliche Hebung der Processus vocales in der Richtung der Trachealachse proportionell mit ihren Längen.

Bis jetzt haben wir eine Stellung der Stimmbänder vorausgesetzt, wie wir sie in der Luschka'schen Figur (Fig. 3) vorfanden. Das heisst also, dass wir annahmen, der hintere Ansatzpunkt des Bandes stehe höher als der vordere mit Bezug auf die Trachealachse. Wir fragen uns ob dieser Fall immer vorkommt, und ob er überhaupt für den Lebenden Geltung hat. Denn es könnten am ausgeschnittenen Kehlkopfe die Verhältnisse anders sein als in vivo. Ein einfaches Raisonnement genügt, um diese Frage zu lösen.

Es gibt ausser dem Höherstehen des hinteren Ansatzpunktes noch zwei Möglichkeiten. Denn erstens könnte dieser Punkt gleich hoch 
stehen wie der vordere Ansatz, zweitens könnte er auch niedriger stehen. Wir haben die drei Fälle in Fig. 5 vorgestellt und sie durch die Zeichen $I, I I$ und $I I I$ unterschieden. Die vorderen Ansatzpunkte $a, a^{\prime}$ und $a^{\prime \prime}$ wurden als Mittelpunkte genommen für Bogen, die mit den Stimmbandlängen als Radius gezogen wurden. Die zugehörige Trachealachse und deren Verlängerungen werden durch die Linien $c d, c^{\prime} d^{\prime \prime}$ und $c^{\prime \prime} d^{\prime \prime}$ vorgestellt.

Wenn der hintere Ansatzpunkt des Stimmbandes $b$ im Fall $I$ sich hebt, so wird sein Abstand von $a$ grösser, senkt $b$ sich aber der Trachealachse entlang, so wird sein Abstand von $a$ verkürzt. Im Fall $I I$ wird das Verschieben von $b^{\prime}$ auf der Achse $c^{\prime} d^{\prime}$ sowohl nach oben wie nach unten eine grössere Entfernung von $a^{\prime}$ hervorrufen. Im Fall $I I I$ dagegen führt die Hebung von $b^{\prime \prime}$ in der Richtung $b^{\prime \prime} d^{\prime \prime}$ eine Verkürzung, eine Senkung von $b^{\prime \prime}$ in der Richtung $b^{\prime \prime} c^{\prime \prime}$ eine Verlängerung der Linie $a^{\prime \prime} b^{\prime \prime}$ herbei.

Die Hebung des Punktes $b$, der dem Processus vocalis entspricht, wird durch die Exspiration, die Senkung durch die Inspiration bewirkt. Die Streckung des Stimmbandes kommt also im Fall $I$ nur bei der Ausatmung, im Fall $I I$ bei der Aus- und Einatmung und im Fall III nur bei der Einatmung zustande. Die laryngoskopische Untersuchung hat uns gelehrt, dass nur das Verhalten wie im ersten Falle zutrifft und die Verhältnisse wie im Fall $I I$ und $I I I$ geradezu pervers genannt werden müssen. Wir formulieren unseren Schluss also folgendermaassen:

Beim Menschen muss der Processus vocalis immer höher stehen als der vordere Ansatzpunkt des Ligamentum vocale mit Beziehung auf die Trachealachse.

Noch bleibt zu ermitteln, wieviel der Processus vocalis höher steht. Werfen wir einen Blick auf Fig. 3, so ist es einlenchtend, dass dieses Wieviel seinen Ausdruck findet in der Grösse des Winkels $d b c$. Man bekommt den nämlichen Winkel, wenn man durch $a$ eine Horizontale auf der Achse der Trachea $c e$ zieht, und $b$ mit $a$ durch eine Gerade vereinigt. Wir bemerkten schon, dass die Abstandsvergrösserung von $a$ und $b$ und also das Strecken des Stimmbandes durch diesen Winkel bestimmt wird. Umgekehrt würde man also aus den Vorgängen bei der Streckung (ohne Dehnung) den Winkel berechnen können. Am ausgeschnittenen Kehlkopf scheint mir das beschwerlich, da die Achse der Trachea dabei nicht mehr fix ist. In vivo ist dagegen das Messen der Hebung des Processus 
vocalis ohne Blosslegung des Kehlkopfes sehr schwer, wie wir schon betonten. Wir brauchen aiso ein anderes Maass. Dieses ist sehr wahrscheinlich zu finden in der Grösse der Exspirationsbewegung, welche die Anspannung bewirkt. Diese aber lässt sich bestimmen nach dem zur Geltung kommenden Exspirationsdrucke. Man musste dann versuchen, laryngoskopisch oder stroboskopisch den Augenblick zu bestimmen, wo die Streckung des Stimmbandes vollendet ist, die Dehnung aber noch nicht angefangen hat. Zugleich hätte man manometrisch den Exspirationsdruck zu messen. Besser scheint mir aber noch der entgegengesetzte Weg, nl. auszugehen von der Stimmbanddehnung und diese herabzumindern, bis nur noch Streckung besteht.

Wenn einmal die Chorda vocalis angespannt ist, so kann sich der Processus vocalis nicht mehr unabhängig vom Schildknorpel weiter erheben. Solange die Rigidität der Befestigung des Aryknorpels noch aushält, braucht dieser Knorpel aber nicht von der Stelle zu rücken. Es erfolgt aber fürs weitere eine Dehnung des Stimmbandes, die den Processus in der Richtung des vorderen Ansatzes des Ligaments zieht. Dieser Gegenzug wächst fortwährend an, je mehr die Hebung fortschreitet. Es muss also ein Moment kommen, wo er der genannten Rigidität die Wage hält. Jetzt übernimmt auch der. Processus vocalis die Rolle als fixer Punkt, so dass das Stimmband als stillstehend betrachtet werden muss. Bei noch weiterer Hebung hat der Aryknorpel nachzugeben.

Form und Art der Bewegung, die nachher erfolgen muss, werden bestimmt durch die Befestigungsweise dieses Knorpelstucks. Am besten können wir uns davon eine Vorstellung bilden mittelst einer Hintenansicht des Ringknorpels. Wir reproduzieren zu diesem Zwiecke eine solche aus Luschka's Kehlkopf (Fig. 6). Wie wir sehen, ist die Spitze des Aryknorpels abgestumpft und nach hinten gebogen. Sie trägt einen kurzen, kegelförmigen, biegsamen Knorpelaufsatz, die Cartilago corniculata s. Santorini. Dieser Knorpel ist durch zellenhaltiges Fasergewebe mit dem Giessbeckenknorpel verbunden. Zuckerkand l, dem wir bei dieser Beschreibung folgen, spricht statt von verbunden von geschieden und nennt die Selbständigkeit des Santorinischen Knorpels scheinbar. Die Spitzen dieser Knörpelchen sind beiderseits auf eigentümliche Weise miteinander verbunden. Jede inseriert nämlich an einem Bändehen, und diese beiden Bändchen vereinigen sich in ihrem weiteren Verlaufe und haften 
am oberen Rande des Ringknorpels. Nicht selten findet man inmitten der gabelförmigen Verbindung ein Knorpelstückchen, die Cartilago interarytaenoïdea. Das so gebildete Ligamentum jugale ist ziemlich straff; S a n to r in i gebraucht die Bezeichnungen „alba, firma, teretia corpora ligamentis similia" für dessen beiden Schenkel. Es muss sich also einem Auseinanderweichen der Spitzen der Aryknorpel widersetzen. Diese Apices müssen daher als relativ fix betrachtet werden mit Bezug auf eine Bewegung lateralwärts.

Der Giessbeekenknorpel hängt andererseits durch ein Gelenk mit dem Ringknorpel zusammen. Der Kapsel dieser Articulatio crico-

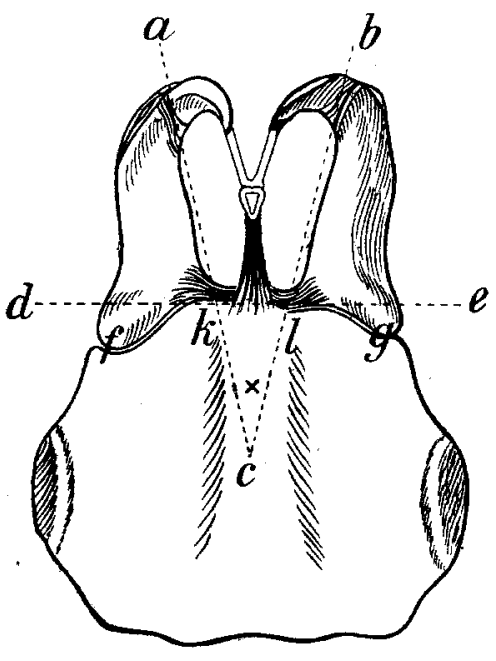

Fig. 6. arytaenoidea ist schlaff und nur an einer Stelle dureh ein Seitenband, das Ligamentum triquetrum, verstärkt. Dieses entspringt am oberen Rande des Ringknorpels, und zwar nach innen vom Gelenkkopfe, und inseriert teils an der hinteren Fläche des Aryknorpels, teils an der Innenseite der höckerig aufgetriebenen Wurzel des Processus vocalis. Da die Insertionslinie eine viel grössere Länge als die Ursprungsstelle besitzt, acquiriert das genannte Band die Form eines Färhers. Diese Ursprungsstelle muss als ein indirekter fixer Punkt des Aryknorpels betrachtet werden. Wir können uns eine Achse denken, welche durch die beiden fixen Punkte (das relative und das indirekte) gelegt ist, die wir baben kennen lernen. Die Achsen von beiden Seiten schneiden einander etwa in der Mitte der Hinterfläche des Ringknorpels unter einem Winkel, den wir als Arywinkel bezeichnen können. Eine Vergrösserung dieses Winkels wird durch das Ligamentum jugale angehalten. Bei der Hebung des Ringknorpels kommen an sich keine Momente in Betracht, die eine Verkleinerung des Arywinkels anstreben. Man muss sich denselben bei diesem Vorgange also unverändert denken.

Wir nannten die Urspungsstelle des Ligamentum triquetrum am Ringknorpel einen indirekten fixen Punkt des Aryknorpels, weil er sich 
ausserbalb derselben befindet. Nebenan, im Giessbeckenknorpel selbst, muss man sich einen anderen Punkt denken, wo die Achse aus- oder, wie man will, eintritt. Zwisehen den beiden Punkten zieht sich das Crieo-arytaenoidalgelenk hin.

Man kann sich diesen unteren Achsenpunkt des Aryknorpels mit dem Processus vocalis durch eine Gerade verbunden denken. Verbindet man auch noch den oberen Achsenpunkt am Santorin i'schen Knorpel mit diesem Processus, so hat man sich die mediale Fläche

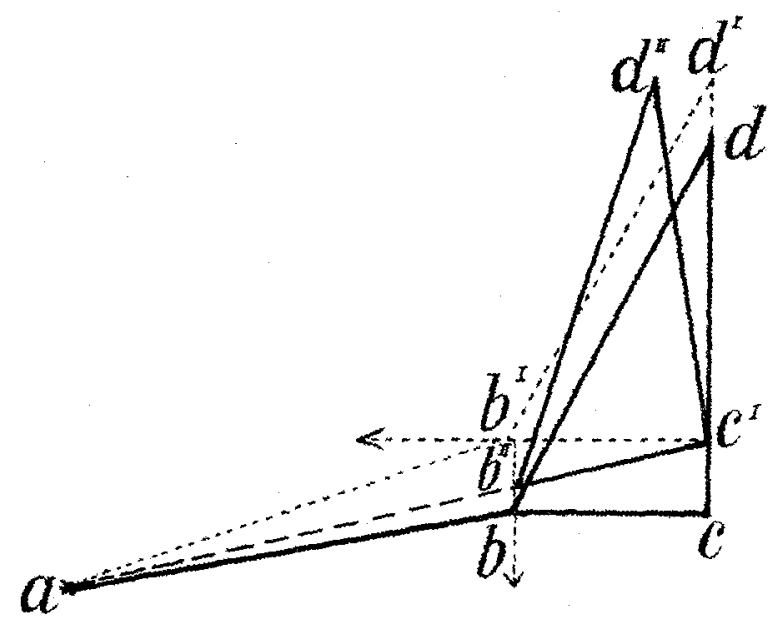

Fig. 7

des Aryknorpels dureh ein Dreieck vergegenwärtigt. Zum leichteren Verständnis denken wir uns den Winkel am unteren Achsenpunkte als einen rechten.

Wie wir gesehen haben, liegt in einer Sagittalebene der vordere Ansatz des Stimmbandes niedriger als der Processus vocalis. Das Verhältnis zum obengenanaten rechtwinkeligen Dreieck kann also vorgestellt werden wie $a b c d$ in Fig. 7 (siehe anch Fig. 3). Hierbei wird angenommen, dass das Stimmband $a b$ gestreckt, nicht gedehnt ist.

Wenn der Ringknorpel sich hebt, so wird das Dreieek $b c d$, das in $b$ o auf ihm rubt, mit gehoben. Die Bewegung findet statt in der Richtung der Trachealachse, die wir als vertikal annehmen. Das Dreieck wird also, wenn sich dem nichts wìdersetzt, parallel an sich selbst hinauf rücken. Solcherweise würde es $z . B$, in die Stellung $b^{\prime} c^{\prime} d^{\prime}$ kommen können. 
Da $a b^{\prime}$ länger ist als $a b$, so müsste hierbei das Stimmband gedehnt werden. Diese Dehnung ist eine Kraft, die $b^{\prime}$ in der Richtung von $a$ zu ziehen sucht. Sie kann entbunden werden in zwei andere Kräfte, deren Richtung wir durch Pfeilchen angegeben haben. Eine Verschiebung von $b^{\prime}$ in horizontaler Richtung nach vorn ist nicht möglich, da die Befestigung des Aryknorpels diese nicht erlaubt. Die Bewegung in der Richtung nach unten wird aber nicht gehemmt. Demzufolge muss der Punkt $b^{\prime}$ sich senken. $c^{\prime}$ aber ist unverrückbar, da er die fixe Verbindung durch das Ligamentum triquetrum vorstellt. Das ganze Dreieck muss sich also um diesen fixen Punkt nach vorn drehen um z. B. die Stellung $b^{\prime \prime} c^{\prime} d^{\prime \prime}$ einzunehmen. Dadurch fällt die Dehnung des Stimmbandes geringer aus, denn $a b^{\prime \prime}$ ist zwar länger als $a b$, aber bedeutend weniger als $a b^{\prime}$.

Der langen Rede kurzer Sinn ist also, dass bei gestrecktem Stimmbande eine Hebung des Ringknorpels zur Vornüberneigung des Aryknorpels führt.

Wenn wir uns diese Bewegung mit Hilfe von Fig. 6 vorstellen, so wird es uns deutlich, dass sie zustande kommen kann bei unverändertem Arywinkel. Sie findet um eine horizontale Achse de statt, die man sich durch die beiden indirekten fixen Punkte gelegt denken kann. Der ganze Rahmen $a k c l b$ bewegt sich so, dass $l$ und $l$ auf der Stelle bleiben, während $a$ und $b$ sich nach vorn und unten drehen. Dagegen muss der Punkt $c$ sich in einer Richtung nach hinten und oben vom Ringknorpel entfernen.

Es leuchtet ein, dass die beiden Processus musculares der Aryknorpel, die wir mit $f$ und $g$ (Fig. 6) angedeutet haben, sich mit Bezug auf die beschriebene Drehung verhalten wie der virtuelle Punkt c. Denn wenn man z. B. $f$ durch Linien mit den beiden fixen Punkten in der Achse $a c$ vereinigt, so wird unterhalb der Achse $d e$ ein Dreieck beschrieben mit $f$ als Spitze, das sich in gleichen Umständen befindet wie $\Delta k c l$.

Bei der Neigung der Aryknorpel nach vorn wird $f$ also versuchen nach hinten und oben auszuweichen. Da dieser Punkt aber beinern verbunden ist mit dem Processus vocalis, so müsste dieser der Bewegung folgen. Das Stimmband, das an ihm verbunden ist, widersetzt sich aber einer Ausweichung von $f$ nąch hinten. Das gilt nicht für dessen Bewegung nach oben. Um das zu verstehen, muss man sich eine Vorstellung bilden vom Crico-arytaenoidalgelenk. 
Wenn man sich den unteren Achsenpunkt eines Aryknorpels und seinen Processus vocalis nebst dem Processus muscularis durch gerade Linien untereinander verbunden denkt, so repräsentiert das gebildete Dreieck die Gelenkfläche dieses Knorpels. Diese wird gequert durchdie derselben entsprechenden Gelenkfläche des Ringknorpels. Zuckerkandl sagt dazu (Heymann's Handb. Bd. 1 [1], S. 66) folgendes: „Partes constituentes der Articulatio crico-arytaenoïdea sind je ein am oberen Rande der Ringknorpelplatte aufsitzender Gelenkkopf und die Basis des Aryknorpels, welche die Gelenkpfanne trägt. Der Gelenkkopf bildet einen elliptisch konturierten, konvex vorspringenden Körper mit wulstig aufgeworfenem, dorsal überhängendem Rande. Infolge der Ringform des Knorpels und seiner allmählichen Höhenzunahme in der Richtung von vorn nach hinten fällt der konvexe Gelenkkörper gleich einer schiefen Ebene ab. Die längere Achse der Gelenkfläche ist, dem Laufe des oberen Ringknorpelrandes folgend, schräg, die kürzere mehr frontal gestellt."

Die Pfanne des Gelenkes beginnt knapp hinter dem Processus vocalis und nimmt die ganze Länge der Aryknorpelbasis für sich in Anspruch, woraus hervorgeht, dass der Processus muscularis an der Pfannenbildung mitbeteiligt ist. An der Cavitas glenoïlalis ist die längere Achse mehr frontal, die kürzere schräg gelagert. Es kreuzen sich demnach die langen Achsen der beiden Gelenkbildner. Bei Luschka finden wir noch folgendes: „Die Kontaktflächen der Cricoarytaenoidalgelenke stimmen in der Grösse unter sich nicht überein, so dass sie sich in keinem Moment der Bewegung allseitig berühren. Der elliptische, zylindrisch gewölbte, von der oberen Ecke der Platte des Ringknorpels sehr steil lateralwärts abfallende Gelenkkopf besitzt durchschnittlich eine der abfallenden Richtung des oberen Randes des. Ringknorpelbogens folgende Länge von $6 \mathrm{~mm}$, während der gerade, mit diesem sich kreuzende Durchmesser sich auf $3 \mathrm{~mm}$ beläuft. Die $5 \mathrm{~mm}$ lange und im Maximum $4 \mathrm{~mm}$ breite Kontaktfläche des Giessbeckenknorpels besitzt die Gestalt eines Ovales, dessen spitzes Ende nach vorn und innen, das stumpfe nach aussen und hinten schaut, dessen Längsachse sich daher mit jener des Gelenkkopfes der Cartilago cricoïdea unter einem mehr oder weniger spitzen Winkel kreuzt. Diese Kontaktfläche ist konkav, stellt jedoch kein Segment einer Hohlkugel, sondern eine Schraube dar, so dass durch die Artic. crico-arytaenoïleae schraubenartige Bewegungen ausgeführt werden können.

E. Pfiüger, Archiv für Physiologie. Bd. 123. 
Mit Hilfe der gewonnenen Vorstellung wird untenstehende Fig. 8 erklärlich sein. Wir haben eine ziemlich weit geöffnete Glottis vorausgesetzt, da die Bewegung, die wir beschreiben, Beziehung hat auf den Anfang einer Exspiration. Der Stand der Artikulationsfläche des rechten Aryknorpels wird durch das Dreieck $a b c$ vorgestellt, wobei $a$ den unteren Achsenpunkt, $b$ den Processus museularis und $c$ den Processus vocalis repräsentiert. Wir nehmen $b c$ an als Längsachse von der Facies glenoildalis des Giessbeckenknorpels. Diese wird gekreuzt durch die Längsachse der Gelenkfläche des Ring-

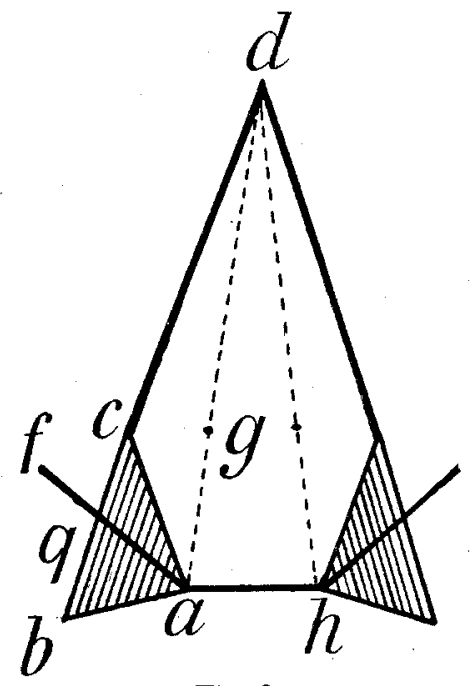

Fig. 8. knorpels, die durch die Linie $a f$ angedeutet wird.

Wenn der Ringknorpel sich weiter hebt, nachdem das Stimmband $d c$ schon gestreckt ist, so versucht der Punkt $c$ unter dem Einflusse des Gegenzuges zurückzubleiben. Der Punkt $a$ muss aber weiter mit, da er fest mit dem Ringknorpel verbunden ist. Solcherweise kommt $a$, wie wir es in Fig. 7 skizziert haben, mit Beziehung auf $c$ höher zu stehen. Der Punkt $b$ kann, wie wir schon bemerkten, nicht nach hinten ausweichen, aber sich wohl heben, während $c$ als relativ fixer Punkt funktioniert. Die Achse $b c$ muss dann sozusagen, nach vorn überkippen. Diese Achse ist aber ebensowenig wie der ganze Aryknorpel aktiv, sie wird also umgekippt. Die Kraft, die das bewirkt, rührt her von dem Aufsteigen des Ringknorpels und deshalb von der Hebung der Gelenkachse af. Sie wird übermittelt durch den Kreuzungspunkt der beiden Achsen $q$. Wenn aber $q$ aufsteigt und $c$ zurückbleibt, so muss der Abstand $q c$ grösser werden; dann aber muss $b q$ kleiner werden. Da die Achse $a f$ sich parallel an sich selbst hebt, behält sie ihre Richtung bei. Die Verkleinerung von $b q$ bedeutet also ein Abgleiten von $b$ in der Richtung von $c$. Da der Punkt $a$ fix ist, muss $b$ und damit auch der Processus vocalis $c$ sich um $a$ als Mittelpunkt nach der Mitte der Stimmritze hin drehen. Noch einfacher stellt man sich die Sache 
vor, wenn man in Betracht zieht, dass die Entfernung von $a$ und $d$ grösser werden muss wenn der erste Punkt sich hebt, während der zweite fix bleibt. Diese Vergrösserung erfordert eine Streckung der gebrochenen Linie $a c d$, und also eine Bewegung von $c$ in der Richtung von $g$.

Die Facies glenoidalis, die wir durch $a b c$ vorgestellt haben, wird dem Gesugten nach in linearer Richtung gehoben, während sie sich zugleich dreht. Das Produkt einer linearen Bewegung mit einer Drehung ist eine Schraubenbewegung. Wie wir sahen, hat Luschka diese Art von Bewegung schon aus der Form der Gelenkfläche abgeleitet.

Neigung und Drehung des Aryknorpels konnten auf ganz bestimmte Weise aus der Hebung des Ringknorpels abgeleitet werden. Wir glauben uns darum berechtigt anzunehmen, dass zwischen beiden ein mathematisches Verhältnis besteht. Dann aber muss man annehmen, dass die Glottisweite in direkter Beziehung steht zur Hebung der Trachealachse und solcherweise zur Tiefe der Exspiration. Je tiefer die Ausatmung, je enger die Stimmritze. Die Natur strebt also keinesfalls eine so schnell mögliche Ausströmung der Luft aus den Lungen an. Demgegenüber besitzt sie in der Glottis eine Vorrichtung, die gesetzmässig diese Ausströmung verzögert. Die Natur käme also mit sich selbst in Widerspruch, wenn die Ausatmung nur den Zweck hätte, Exkretionsprodukte fortzuschaffen. Nur wenn man annimmt, wie wir es in "die Nase als Luftweg" schon taten, dass der Wert einer Exspiration auch bestimmt wird durch die Zeit, die sie anhält, wird uns die Glottisvorrichtung erklärlich. Da eine tiefere Exspiration zugleich eine grössere Verzögerung mit sich bringt, kommen die so wechselnden Kräfte stets zu ihrem Rechte. - Graphisch muss sich diese Regulierung in der Form der Exspirationskurve kundgeben. Die Neigung des absteigenden Schenkels der Respirationskurve wird, wenigstens hauptsächlich, durch die Glottis beherrseht und in dieser Beherrschung gipfelt ihr physiologischer Nutzen.

Jetzt steht uns die Analogie zwischen Nasenventil und Glottis klar vor Augen. Werfen wir noch einen Blick zurück auf Fig. 1, so erscheinen uns die Klappenapparate an den Zugängen zum oberen Luftraume höchst rationell. Wie treue Pförtner überwachen sie einerseits die eintretende Aussenluft, andererseits die austretende Lungenluft. Aber nicht nur Pförtner, auch Herrscher sind sie. Es möge der 
Brustkasten toben wie er wolle, er bleibt den Gesetzen der beiden Apparate unterworfen. Weder, die Inspiration noch die Exspiration kann willkürlich schalten. Zwar behaupten sie beim Einsetzen ihre Selbständigkeit, aber sofort greifen die Klappenvorrichtungen ein, um ihnen die Gesetze für ihr zeitliches Verhalten vorzuschreiben. Solcherweise wird einer Verschwendung von Kraft vorgebeugt. Diese Fürsorge ist eine plysiologische Notwendigkeit, denn eine Bewegung, die sich Tag und Nacht etwa tausendmal pro Stunde wiederholt, lässt auch eine kleine Verschwendung nicht zu ohne schwere Folge.

Die bis jetzt beschriebene Bewegung des Aryknorpels wurde von uns abhängig gemacht von dem Gegenzug, der durch das gestreckte Stimmband ausgeübt wurde. Dieser Gegenzug wird bemessen durch den Widerstand, den das elastische Band seiner Dehnung entgegensetzt. Demgemäss strebt dieses Band der Stellung zu, wobei seine Dehnung am geringsten ist.

Betrachten wir in Figur 8 die gebrochene Linie $a c d$, so leuchtet es ein, dass $d c$ sich verkürzen kann, wenn der Processus vocalis c sich nach der Mittellinie der Glottis hin bewegt. Wenn aber die gebrochene Linie ganz gestreckt und $c$ also in $g$ angekommen ist, ist das Band $d g$ anch am kürzesten. Wenn $g$ sich noch weiter der Mittellinie nähern würde, so müsste die Chorda $d g$ wieder anfangen sich zu verlängern. Auch wenn das Stimmband die Bewegung aktiv leitete, so würde beim Erreichen der Stellung $d g$ seine höchste Leistung vollbracht sein. Die Theorie, die die Spannung der Stimmbänder auf ihre eigene Kontraktion zurückführen will, könnte aber auch nur bis an diese Grenze gelten, da eine weitere Annäherung von $d$ und $g$ auch bei stärkster Kontraktion nicht mehr möglich ist. Jedenfalls bedeutet also die Stellung $d g$ den Endpunkt der Anspannung des Stimmbandes.

Die Hebung des Ringknorpels dem Schildknorpel gegenüber ist nicht unbeschränkt. Die anatomischen Verhältnisse schreiben dieser Bewegung ihre Grenze vor. Diese findet sich abgezeichnet im Cricothyreoïdalgelenk. Wenn die Bänder dieses Gelenkes eine weitere Verschiebung des Ringes nach oben nicht mehr gestatten, hat auch die respiratorische Verengung ihren höchsten Punkt erreicht. Zwar kann die Trachea sich noch weiter heben, aber dann nimmt sie den Schildknorpel mit. Die beiden Ansatzpunkte des Stimmbandes werden somit in gleicher Weise gehoben und ändern ihre gegenseitige Stellung nicht mehr. 
Solange die Glottisverengung ohne Vermittelung von Muskeln vor sich geht, können wir sie automatisch nennen. Die grösstmögliche automatische Verengerung muss zusammenfallen mit dem Glottisstand, den wir durch die punktierte Linie in Fig. 8 angegeben haben. Es wäre möglich, dass eine weitere Hebung des hinteren Ansatzpunktes des Stimmbandes noch eine Dehnung dieses Bandes bewirkte, aber ein weiteres Hineinrücken über $g$ hinaus ist auf automatischem Wege nicht denkbar.

Das Analogon dieser Position der Stimmlippen ist auch am Nasenventil zu finden. Denn die höchste Leistung der automatischen Funktion ist hier an die tiefste Inspiration gebunden. Die Grenze hierfür ist in der Dehnbarkeit der Membran zu suchen (siehe Fig. 2 I.).

Gleich wie am Naseneingange finden wir auch an der Glottis Muskeln, die imstande sind den automatischen Vorgang zu fixieren und zu verstärken. In erster Linie kommt hierfür der Musculus Crico-thyreoïdeus in Betracht.

Zweifellos dient dieser Muskel dazu, um den Abstand zwischen Ring- und Schildknorpel zu verkleinern. Fraglich ist es nur, in welcher Richtung diese Funktion zur Äusserung kommt. Es könnte die untere Hälfte des Kehlkopfs zur oberen oder umgekehrt die obere zur unteren herangezogen werden. Die dritte Möglichkeit wäre, dass beides zugleich stattfände und die beiden Hälften also einander näherten.

Viele glauben, dass der Musculus crico-thyreoïdeus der eigentliche Stimmbandspanner sei. Da die Anspannung, wie erörtert wurde, auf eine Hebung des Ringknorpels zurückzuführen ist, so sollte die Funktion dieser Muskel darin bestehen, diese Hebung zu erwirken. Nach der von uns gegebenen Darstellung ist das Aufsteigen des Ringes nur eine Teilerscheinung von der nämlichen Bewegung von Luftröhre und Lungen. Man kann sich beschwerlich vorstellen, dass die kleinen Crico-thyreoïdei zu diesem Kraftaufwande imstande sein würden. Mit gutem Willen könnte man noch annehmen, dass sie den Ringknorpel an sich heben könnten. Das aber würde ein Ausziehen des ziemlich straffen Bindegewebes erfordern, womit der Knorpel am ersten Trachealringe verbunden ist. Bei schnell aufeinanderfolgenden Stimmbandspannungen wie beim Sprechen und Singen würde diese Arbeit durch das Muskelchen viele Male kurz nacheinander wiederholt werden müssen. Das klingt zu unwahrscheinlich. 
Ich hoffe, dass man meinen Zweifel an solch einer Funktion der ziemlich winzigen Muskeln begreiflich finde. Damit ist aber nicht ausgeschlossen, dass ihre Arbeit die Hebung des Ringknorpels etwas fördert; auch ein Kind kann eine Maschine drehen helfen.

Viel eher kann man sich vorstellen, dass die Crico-thyreoïdei den Schildknorpel herunterziehen. Die Dehnbarkeit der Membrana und der Ligamenta thyreo- hyoïdea, womit dieser Knorpel am Zungenbein aufgehängt ist, würde eine geringe Bewegung nach unten zulassen. Der Musculus thyreo-byoïdeus braucht dafür auch kein bedeutendes Hindernis zu sein. Es wäre also möglich, dass diese Wirkung dann und wann durch die Kontraktion der Cricothyreoïdei hervorgerufen würde. Solcherweise müssten diese Muskeln als Depressoren des Schildknorpels aufgefasst werden.

Diese Depression darf aber nicht gelten als die Hauptfunktion der Stimmbandspannung, wie wir schon früher betont haben. Sie muss aber von Bedeutung sein für die Fixation des vorderen Ansatzpunktes des Stimmbandes, die eine conditio sine qua non für diese Anspannung ist.

Die Musculi sterno-thyreoïdei wirken im gleichen Sinne. Sie sind viel grösser und stärker als die Crico-thyreö̈dei, aber sie überbrücken nicht direkt gleichwie diese letzten die Spalte zwischen den beiden Hälften des Kehlkopfs. Man ist geneigt, die Hauptfunktion bei der Fixierung des Schildknorpeis den grossen Sternothyreoïdei zuzuschreiben und die Wirkung der Crico-thyreoïdei mehr in direkte Verbindung mit der überbrückten Spalte zu bringen.

Am liebsten stellen wir uns die Sache auf folgende Weise vor: Die Zwerchfellbewegung hebt respiratorisch den Ringknorpel, spannt die Stimmbänder und bestimmt die Weite der Stimmritze. Der Gegenzug, nötig zur Fixierung des Schildknorpels, wird hauptsächlich geliefert durch die Sterno-thyreoïdei. Da das Sternum sich bei der Exspiration senkt, genügt schon ein Tonus dieser Muskeln, den Schildknorpel mit nach unten zu ziehen. Die beiden Partes constituentes des Kehlkopfs werden also bei der Ausatmung in entgegengesetzter Richtung bewegt.

Die Verschiebung ist gebunden, wie schon bemerkt wurde, an das Gelenk zwischen Schild- und Ringknorpel und findet also ihre Grenzen in der Beweglichkeit dieser Verbindung. Das Gelenk ist aber sehr klein und liegt ziemlich weit nach hinten. Wenn man 
sich eine vertikale Ebene durch die beiderseitigen Gelenke gelegt denkt, so würde diese ungefähr die Processcus vocales treffen.

Die Spalte an der Vorderseite des Kehlkopfs zwischen Schild- und Ringknorpel würde noch Bewegungen zulassen, und dadurch die erreichte Stimmbandspannung unsicher gestalten. Wenn sich aber die Museuli crico-thyreoïdei kontrahieren, so ziehen sie die beiden Kehlkopfhälften an der Vorderseite nacheinander zu. Sie fördern sowohl die Hebung des Ringes wie die Senkung des Schildknorpels. Man muss aber annehmen, dass sie zu schwach sind, um eine weitere Annäherung von einiger Bedeutung zu erwirken. Ihre Funktion muss also vielmehr als eine Fixierung des schon bestehenden Zustandes aufgefasst werden. Sie sichern solcherweise die Stellung der vorderen Ansatzpunkte der Stimmbänder. Wenn sie nicht fortwährend kontrahiert bleiben, sondern rhythmisch erschlaffen, so muss die Unsicherheit in der Stimmbandspannung abwechselnd hervortreten. Vielleicht kann hieraus das tremolo der Stimme erklärt werden.

Wie von selbst betreten wir bei der Besprechung der Musculi crico-thyreoïdei das Gebiet der phonetischen Funktion des Kehlkopfs. Die antomatische Funktion, wovon bis jetzt die Rede war, braucht die Fixierung des Glottisstandes nicht. Vielmehr hat man hierbei eine labile Mittelstellung der Glottis zu erwarten, die sich leicht den respiratorischen Druckschwankungen anpassen kann. Dass diese Anpassung wirklich besteht, lässt sich laryngoscopisch manchmal deutlich genug verfolgen. Eine Fixierung der Glottis könnte diese Anpassung nur erschweren und gehört also nicht mehr in den Rahmen der automatischen oder respiratorischen Kehlkopffunktion. Deshalb kann der Musculus crico-thyreoïdeus auch nicht als Atmungsmuskel aufgefasst werden, gleichwie der Sternothyreoïdeus. Mit seiner Kontraktion tritt der Kehlkopf in einer neuen Rolle auf.

Wenn die bejden Hälften des Larynx hinten durch die Cricothyreö̈dgelenke, vorn durch Muskélwirkung in ihrer Verschiebung gehemmt sind, machen sie weitere Bewegungen zusammen mit. Der Kehlkopf kann sich zwar noch heben und senken, aber nur als Ganzes. Eine weitere Anspannung der Stimmbänder kann nicht mehr stattfinden, weil ihre vorderen und hinteren Ansatzpunkte sich nicht mehr voneinander entfernen. Die Glottisstellung, die beim Anfang der Kontraktion der Crico-thyreoïdei bestand, ändert sich daher nicht mehr infolge der Respirationsbewegungen. Die Glottis- 
verengerung, wie sie antomatisch hervorgebracht wurde, bleibt also auf ihrem höchsten Punkte stehen.

Dieser Zustand der Glottis muss sich vorfinden bei der tiefstmöglichen Exspiration, wenn jede Muskelwirkung ausgeschlossen ist. Beide Bedingungen findet man erfüllt beim Kadaver. Hier haben wir also die höchste Leistung der automatischen Verengung der Stimmritze zu erwarten. Wir wissen dass an der Leiche eine ziemlich starke Annäherung der Stimmlippen an die Mittellinie vorgefunden wird, die seit v. Zi ems en den Namen Kadaverstellung trägt. Diese Stellung muss also nahezu erreicht sein, wenn der Musculus crico-thyreoïdeus anfängt zu funktionieren. Wir sagen "nahezu", da wir es für möglich und selbst für wahrscheinlich halten, dass die Muskelkontraktion die beiden schon anliegenden Kehlkopfhälften etwas genauer anschmiegt und solcherweise die Stimmritze noch um ein weniges verengt. Wir halten das aber für nebensächlich und fassen unsere Meinung zusammen in dem Satz: die Musculi crico-thyreoudei fixieren die Glottis in der Kadaverstellung. Diese Fixierung markiert das Ende der respiratorischen Funktion des Kehlkopfes. Zugleich bildet sie den Anfang einer anderen Funktion von diesem Organe, nämlich die phonetische. Die Kontraktion der Crico-thyreoïdei stellt sozusagen die Brücke dar, die beide Funktionen verbindet. Sie bildet den Übergang von der gewöhnlichen zur tönenden Exspiration. Gleichwie man beim Heben eines Gewichtes an die Zugrolle, das Seil befestigt um zu behalten, was man schon erreicht hat, so sichert die Kontraktion der Cricotbyreoïdei den Effekt der respiratorischen Verengerung. Und ebenso wie das Erreichte mit der Zugrolle der weiteren Hebung zugrunde liegt, so hat die fixierte Kadaverstellung als die Basis der phonetischen Wirksamkeit der Glottis zu gelten.

Von diesem Standpunkte aus sind wir genötigt einen Zusammenhang anzunehmen zwischen dieser Glottisstellung und dem Anfang der Lautbildung. Wir bringen also die Kadaverstellung des Kehlkopfes mit dem Grundton der Stimme in Verbindung.

Da dieser Grundton bei versehiedenen Individuen auch verschieden ist, so müssen wir auch eine Ungleichheit in der Kadaverstellung annehmen. In Wirklichkeit schwankt die Glottisweite bei diesem Zustande auch zwischen ziemlich weit auseinanderliegenden Grenzen. Zum Beweise reproduzieren wir hier eine Figur aus Semon's Artikel in Heymann's Handbuch Bd. 1 (1) S. 640. (Fig. 9.) 
Wie man sieht ähnelt die Kadaverstellung (am wenigsten in maximaler Weite) dem Verhältnisse, wie wir es durch die punktierten Linien in Fig. 8 angegeben haben. Es leuchtet ein, dass die Grösse des Dreieckes $a d h$ sowohl durch die Länge von $a h$ wie durch die Länge von $a d$ bestimmt wird. Beide Längen können wechseln und solcherweise sehr verschiedene Dreiecke gebildet werden. Diese Verschiedenheit kann sowohl Beziehung haben auf die Grösse wie auf die Form des Dreieckes.

Es liegt ausserhalb des Rahmens dieser Arbeit, die physischen Gesetze zu besprechen, die die Tonbildung durch die Glottis beherrschen. Wir können aber nicht umhin, mit Bezug auf die Haupt-

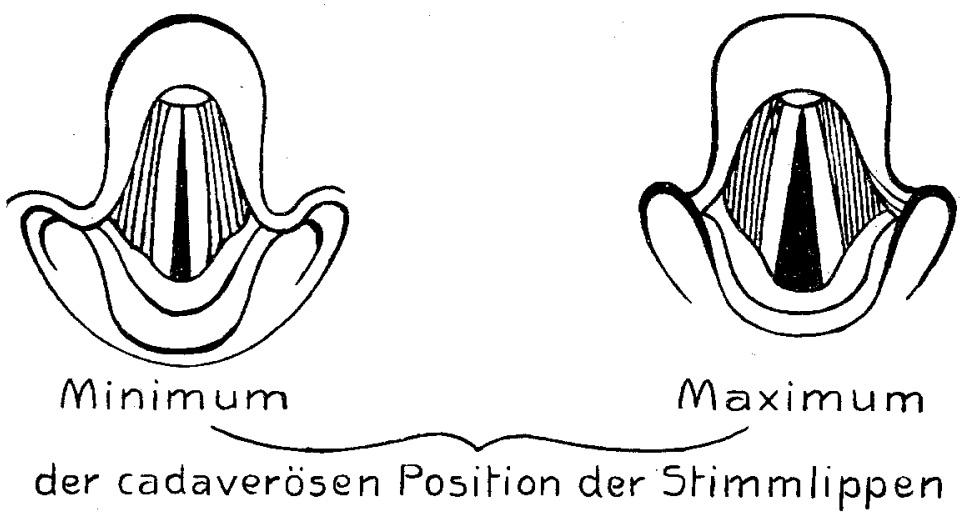

Fig. 9.

sache Stellung zu nehmen. Wenn wir nochmals die Fig. 8 betrachten, so ist es deutlich, dass das Stimmband in der Position $d g$ am kürzesten ist. Das Band ist zwar gestreckt, aber von einer bedeutenden Dehnung kann schwerlich die Rede sein. Das springt noch besser ins Auge, wenn wir einen Blick auf Fig. 7 werfen.

Beim Annähern des Processus vocalis $g$ (Fig. 8) zar Mittellinie ist nur noch eine minimale Verlängerung des Stimmbandes möglich. Und doch ist in dieser Annäherung die Bildung einer ganzen Tonreihe enthalten. Es ist wohl ausgeschlossen, dass diese auf die winzige passive Dehnung des Stimmbandes zurückzuführen sei. Das nimmt man denn auch nicht an. Nach dem schon zitierten Satze von Zuckerkandl ist der Musculus vocalis berufen, die schon vorher gedebnte Stimmmembran anzuspannen. Ich vermute, dass mit dieser vorherigen Dehnung der Zustand gemeint wird, den wir durch die 
punktierte Linie in Fig. 8 vorgestellt haben. Der Musculus thyreoarytaenoideus internus oder $M$. vocalis wie Zuckerkandl ihn nennt, läuft neben und parallel an der Chorda $d g$. Mir ist es unbegreiflich, was eine Kontraktion dieser Muskel noch an dieser Stimmbandstellung ändern kann. Denn in dieser Stellung ist die Muskel am kürzesten und hat er also seine höchste Leistung erreicht. Eine weitere Verengerung der Stimmritze muss wieder eine wenn auch unbedeutende Verlängerung der Muskel bewirken. Wie das aber mit einer aktiven Anspannung zu vereinbaren ist, ist mir vollkommen unklar.

Man kann sich denken, dass der M. vocalis die Chorda zur Stellung $d g$ hinzieht, wenn diese in der einen oder der anderen Richtung davon abweicht. Solcherweise würde er dann in gleichem Sinne arbeiten wie die Elastizität der Chorda selbst. Seine Kontraktion würde dann aufzufassen sein als das Zufügen einer aktiven Elastizität zu der passiven der Chorda. Das scheint uns eine nützliche Aufgabe, die gross genug ist für dieses Muskelchen. Aber damit verdient es den Namen Stimmuskel nicht. Wenn das Stimmband in der Kadaverstellung angekommen ist, hat es seine Arbeit schon vollbracht; seine Wirksamkeit liegt also ganz im Gebiete der respiratorischen Funktion des Kehlkopfs. Der sogenannte M. vocalis muss deshalb als Respirationsmuskel aufgefasst werden. Mit der Tonbildung hat er nur insoweit zu schaffen, dass er gleich wie der Crico-thyreoildeus die Glottis in der Kadaverstellung fixieren hilft. Auch er bildet solcherweise eine Brücke für den Übergang von der gewöhnlichen zur tönenden Exspiration.

Uns erübrigt nur, die Tonbildung ganz und gar von Weite und Form der Stimmritze abhängig zu stellen. Reine Töne erfordern aber eine gewisse Festigkeit von den Rändern dieser Öffnung. Indem der Musculus internus dieselbe besorgt, ist er von grundlegender Bedeutung für die phonetische Funktion des Kehlkopfes, ohne zu den Tonbildnern gerechnet werden zu dürfen.

Die laryngoskopische Untersuchung lehrt uns, dass ein höherer Ton einer engeren Stimmbandritze entspricht. Das muss auch schon für den Grundton gelten. Wenn unsere Voraussetzung über den Zusammenhang der Kadaverstellung mit dem Grundton richtig ist, werden wir z. B. bei Frauenkadaver fast regelmässig eine kleinere Stimmritze finden. Dabei hat man nicht allein mit der Länge der Stimmbänder zu rechnen. Der Bemerkung von 
Moritz Sehmidt ${ }^{1}$ ): "ich habe Tenoristen untersucht, welche verhältnismässig sehr lange Stimmbünder, und Bassisten, die auffallend kurze hatten" wird wohl jeder Laryngologe beipflichten. Aus Fig. 8. ist ersichtlich, dass auch der Abstand zwischen den Aryknorpeln in Betracht gezogen werden muss. Wie schon bemerkt, kann bei gleicher Grösse auch die Form der dreieckigen Spalte wechseln, was vielleicht für das Timbre der Stimme durchschlaggebend ist.

Beim gewöhnlichen Gebrauch der Stimme kommt der tiefst erreichbare Ton wohl nur ausnahmsweise (dagegen wohl beim Husten) zur Geltung. Wir müssen also annehmen, dass die phonetische Funktion der Glottis selten Beziehung hat auf die Kadaverstellung. Als Regel hat man eine Stimmritze zu erwarten, die enger ist. Diese weitere Verengerung: beruht auf Muskelwirkung und hat mit der Respiration nichts mehr zu schaffen. Sie wird erwirkt durch eine willkürliche Weiterdrehung des Processus vocalis nach der Mittellinie zu. Diese Aufgabe wird durch den Glottisschliesser, den Musculus crico-ary taenoideus lateralis, erledigt.

Diese Muskel greift am Processus muscularis (Fig. 8b) an und dreht den Aryknorpel weiter in der Richtung, die schon automatisch gefolgt wurde. Der Processus vocalis $c$, der sich in Stellung $g$ (Kadaverstellung) befand, nähert sich infolgedessen noch mehr der Mittellinie. Am Ende berühren die Processus der beiden Seiten einander, sodass auch die Chordae aneinander liegen. Angeblich wird bei dieser Bewegung der Processus vocalis noch wieder ein wenig gesenkt. Damit muss auch die Dehnung des Stimmbandes (siehe Fig. 4) geringer ausfallen.

Bis jetzt kam keine Bewegung ins Spiel, die den Abstand ah (Fig. 8) zu verkleinern suchte. Das braucht auch noch nicht der Fall zu sein, wenn die Processus vocales einander berühren. Es erübrigt dann zwischen den Stimmlippen noch ein Raum, der $a h$ als Basis und den Vereinigungspunkt der beiden Processus als Spitze hat. Man ist gewöhnt, demselben den Namen von Glottis respiratoria zu geben, obwohl jeder Grund für diese Benennung fehlt.

Die Annäherung der beiden Punkte $a$ und $h$ wird bewirkt durch Muskeln, die an der Hinterseite zwischen den beiden Aryknorpeln ausgespannt sind. In erster Linie steht hierbei der Musculus arytaenoïdeus transversus. Wenn man sich die Wirkung dieses Muskels

1) M. Schmidt, Die Krankheiten der oberen Luftwege. 1. Ed. S. 48. 
an Fig. 6 vergegenwärtigt, so sieht man ein, dass es möglich wäre, dass er die Punkte $k$ und $l$ nacheinander zu ziehen könnte, obne dass der Arywinkel sich zu ändern braucht. Hierbei würden die Achsen $a b$ und $b c$ sich parallel an sich selbst nach der Mitte hin verschieben, während ibr Schneidepunkt $c$ entsprechend böher zu liegen käme.

Wir glauben aber, dass eine isolierte Kontraktion des Transversus wohl nicht vorkommt, und dass der Konstruktor vestibuli immer zugleich mit in Wirkung tritt. Die gekreuzten Bündel dieses letzten Muskels sind geeignet, die Spitzen der Aryknorpel aneinander zu schmiegen und soleherweise den Arywinkel zu schliessen. Hiermit ist der vollständige Verschluss der Glottis erreicht.

Am Nasenventil fanden wir das Analogon der bis jetzt beschriebenen Muskelwirkung vor. Auch da fanden wir im Anschluss an die automatische Verengerung einen Muskelapparat, um diese noch weiter durchzuführen. Wenn wir die aktive Verengerung des Naseneinganges näher betrachten, schrieben wir in „das Spiel der Nasenflügel", so sehen wir, dass der ganze untere Nasenteil an der Bewegung teilnimmt. Am auffallendsten ist das Absteigen der Nasenflügel und damit auch der Nasenspitze und Septum mobile.

Wir sehen, dass wir uns gleich wie bei der Glottis gedrängt fühlen, die Depression in erster Linie zu nennen. Denn wir haben gesehen, dass nach unserer Vorstellung der Musculus crico-thyreödeus hauptsächlich als Depressor zu gelten hat. Wir vergleichen also diese Kehlkopfmuskeln mit den Depressoren von Nasenflügel und Septum mobile. In beiden Fällen wird die automatisch erreichte Verengerung durch die Depression gesichert.

Wir fanden bei der Nase die Depression begleitet von einer Kompression. Wir vermuten, dass bei der Glottis das nämliche anzutreffen ist.

Die Kompressoren der Glottis sind, wie wir gesehen haben, die beiden Musculi crico-arytaenoïdei laterales und der Musculus interarytaenoideus transversus. Die funktionelle Zusammengehörigkeit dieser drei Muskeln kommt auch in ihrem Faserverlauf zum Ausdruck. Wenn der Processus musculosus keine Unterbrechung darstellte, so würde man die Bündel des Transversus als eine Fortsetzung von denen der Laterales betrachten können. In einer der Figuren von Luschka (Taf. VI Fig. 5) sieht man selbst, wie einige Fasern des Lateralis sich ohne Unterbrechung bis in den Transversus fortsetzen. Wenn dieses für alle Fasern gülte, so würde der M. transversus im nämlichen Verbältnisse stehen zu den beiden Laterales, wie an 
der Nase die Aponeurose zu ihren beiden Kompressoren. So konute man den Transversus gewissermaassen als eine muskulöse Aponeurose der Laterales betrachten.

Der vollkommene Glottisschluss kann natürlich pur kurze Zeit währen. Eine Vorrichtung ist nötig, die der Eröffnung wieder anstrebt. Der autagonistische Vorgang muss zeitlich in eine der Schliessung vollständig entgegengesetzter Reihenfolge stattfinden. Er hat also anzufangen mit der Aufhebung des letzten Aktes des Glottisschlusses. Die Eröffnung muss daher mit der Wiederherstellung des Arywinkels beginnen.

Wir haben gesehen, dass es der Konstriktor vestibuli war, der diesen Winkel aufhob, um die Spitzen der Aryknorpel nacheinander. zu ziehen. Das Entgegengesetzte wird durch den Dilatator vestibuli erwirkt. Dieser Muskel inseriert nicht am Giessbeckenknorpel selbst, sondern an der Membrana quadrangularis, die sich an diesen Knorpel heftet. Ausnahmsweise, jedoch nicht selten, kommt es vor, dass sich sämtliche Fasern dieses Muskels an die Konvexität eines sehr flachen Sehnenbogens ansetzen, weleher zwischen der Mitte des lateralen Randes der Cartilago arytaenoïdea und des Knorpels der Epiglottis ausgespannt ist (Luschka). Solcherweise wird auch die Teilnahme des Kehldeckels an der Glottiseröffnung begreiflich.

Die nächste Aufgabe der antagonistischen Vorrichtung, nachdem der Arywinkel wieder gebildet ist, besteht in dem Auseinanderziehen der unteren Achsenpunkte. Wir brauchen dazu Muskeln, die an der lateralen Fläche der Aryknorpel angreifen und diese lateralwärts ziehen. Hierfür können nur Bündel des Musculus thyreoarytaenoideus externus in Betracht kommen. Der verwickelte Verlauf der Fasern dieses Muskels gibt aber einer anderen Deutung seiner Wirkung Raum.

Nachdem die Aryknorpel lateralwärts gezogen sind, ist die Wirkung des Musculus interarytaenoideus transversus ausgeschaltet. Die Achsen, die den Arywinkel bilden, befinden sich wieder in der Stellung wie sie in Fig. 6 angegeben ist, aber noch immer nach vorn übergebeugt. Den virtuellen Punkt $c$ müssen wir also als noch nach hinten von der Ringknorpelplatte abgewichen denken.

Processus vocales und chordae liegen noch aneinander, aber die sogenannte Glottis respiratoria ist schon formiert. Die nächste Aufgabe der Eröffnung ist die Zurückführung der Stimmbänder bis in die Kadaverstellung. Diese fällt dem Musculus crico-arytaenoïdeus posticus zu. 
Dieser Muskel, kurzweg Posticus genannt, ist der Antagonist des Lateralis oder Glottisschliessers. Seine Kontraktion zieht den Processus muscularis nach hinten und unten. Die Bewegung nach hinten kann frei erfolgen und erwirkt eine Drehung des Aryknorpels, solcherweise, dass der Processus vocalis zurückgedreht wird bis in die Kadaverstellung. Die Bewegung nach unten ist aber nicht möglich, solange der Ringknorpel seine Stellung beibehält. Vorläufig kann also nur von einem Andrücken des Processus muscularis an den Ring die Rede sein. Jetzt ist die Kadaverstellung, wie wir sie früher beschrieben haben, wieder hergestellt.

Am Nasenventil wird die Eröffnung des verkleinerten inneren Nasenloches durch die Hebung des Nasenflügels erzwungen: Der Levator alae nasi tritt da also in der nämlichen Rolle auf wie der Posticus an der Glottis. Wir haben deshalb bei der Vergleichung der beiden Zugänge zum oberen Luftraume, den Posticus als das Analogon des Nasenflügelhebers zu betrachten.

Wir erwähnten in "Das Spiel der Nasenflügel" auch zwei kleine Muskelchen, den M. levator alae nasi proprius anterior und den M. levator alae nasi proprius posterior. Ihr Hauptzweck schien uns zu sein, den Nasenflügel zu steifen und auf diese Weise die Hebung dieses Flügels als Ganzes zu fördern. Die Dilatation des äusseren Nasenloches, die von diesen kleinen Muskeln erwirkt wird, kann also als eine Vorbereitung für die Eröffnung des eigentlichen Nasenzuganges aufgefasst werden. Wir können eine Parallele ziehen zwischen dieser Vorbereitung und der Bildung der Glottis respiratoria bei noch anliegenden Stimmbändern. Sodann können die Dilatatoren des Nasenflügels als Analoga betrachtet werden von den Dilatatoren des Hinterendes der Stimmritze.

Ob wir auch eine Muskulatur am Kehlkopfe anzunehmen haben, die in gleichem Sinne wirkt wie der Musculus procerus an der Nase, ist fraglich. Beachtung verdient jedenfalls folgende Äusserung von Luschka: Dass aber eine eigene auf die aktive Erweiterung der oberen Kehlkopfapertur und des Cavum vestibuli berechnete Muskulatur wirklich als gesetzmässige Bildung existiert, ist schon von Carl Ludwig Merkel mit gutem Grunde eingeräumt worden. Dem seit den Arbeiten Santorini's als Thyreo-epiglotticus bekannten Muskel, welcher mit dem sogenannten Ary-epiglotticus von späteren Autoren nach dem Vorgange Theile's als "Reflektor epiglottidis" zusammengefasst worden ist, hat Merkel, wie mir scheint, mit vollem Rechte die Aufgabe zugeschrieben, die Membrana quadran- 
gularis lateralwärts zuziehen und ihn, weil er vom Schildknorpel hauptsächlich auf jene Grundlage der Plica ary-epiglottica übergreift, "Stratum thyreo-membranosum" genannt.

Die Verhältnisse der Muskelfasern, die unter dem Sammelnamen von Dilatator vestibuli Laryngis zusammengefasst werden, sind ziemlich verwickelt. Wenn hier ein Analogon des Procerus zu finden ist, dann kommt umgekehrt die Frage auf, ob dieser Muskel vielleicht auch als ein Dilatator vestibuli nasi zu gelten hätte.

Die Beschreibung der Eröffnung der Glottis ist jetzt bis an die Kadaverstellung fortgeschritten. Die Glottis wird in dieser Stellung festgehalten, wie wir gesehen haben, durch den Musculus cricothyreoïdeus. Dessen Kontraktion muss nachlassen, bevor eine weitere Eröffnung möglich ist. Wenn dieselbe stattgefunden hat; ist die Einheit des Kehlkopfes aufgelöst, so dass wir wieder mit seinen beiden Hälften zu schaffen haben. Dieser Vorgang markiert das Ende der phonetischen und den Wiederanfang der respiratorischen Funktion des Larynx. Letztere hat aber nun Beziehung auf die Inspiration. Wir haben deshalb zugleich mit einer Hebung des Sternums Rechnung zu schaffen.

Wenn das Brustbein aufsteigt, muss der Musculus sternothyreoïdeus durch die Annäherung seiner beiden Ansatzpunkte erschlaffen. Dadurch kommt der Musculus thyro-hyoüdeus, der bei der vorangehenden Exspiration vielleicht etwas gedehnt war, Gelegenheit, sich zu kontrahieren. Lässt die Kontraktion der Crico-thyreoidei nach, so kann der Schildknorpel unter dem Einflusse des Thyreohyoïdeus nach oben gezogen werden.

Der Ringknorpel folgt dieser Bewegung nicht, da er bis auf eine gewisse Höhe unabhängig geworden ist von der oberen Hälfte des Kehlkopfes. Dagegen steht er unter der Herrschaft von Trachea und Lungen, die sich mit dem Zwerchfell senken. Es ist also in der Inspirationsbewegung selbst eine doppelte Ursache enthalten für das Auseinanderrücken der beiden Kehlkopfhälften.

Eine Hebung des Schildknorpels bedeutet eine höhere Lage für die vorderen Ansatzpunkte der Stimmbänder. Eine Senkung des Ringknorpels bringt dagegen eine niedrigere Lage der hinteren Ansatzpunkte dieses Bandes mit sich.

Vergegenwärtigen wir uns diesen Vorgang an Fig. 3, so ist es deutlich, dass der Winkel $d b c$ kleiner werden muss. Die beiden Ansatzpunkte kommen dadurch mehr auf gleiche Höhe zu liegen mit Bezug auf die Trachealachse. Wenn die Bewegung des Schildknorpels parallel an dieser Achse erfolgt, so nähern sich die Ansatz- 
punkte, so dass das Stimmband erschlaffen muss. Auch wenn der vordere Ansatz auf der Stelle bleibt, muss das Sinken der Processus vocales an sich schon diese Erschlaffung herbeiführen, was ohne weiteres aus Fig. 5 deutlich ist. Jedenfalls dürfen wir annehmen, dass dieser Processus

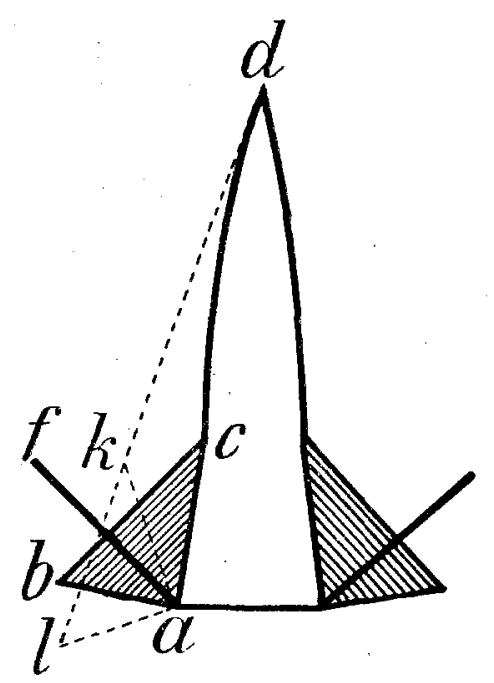

Fig. 10 . nach dem Einsetzen der Inspiration aufhört als fixer Punkt zu fungieren.

Wir stellen uns den Sachbestand vor durch untenstehende Fig. 10. Absichtlich haben wir die Chordae vocales etwas gebogen gezeichnet, um anzudeuten, dass dc nicht mehr als gespannt betrachtet werden muss. Die erste Folge dieses Zustandes muss sein, dass $c$ nicht mehr nach unten gezogen bleibt mit Beziehung auf $a$, wie es durch die Fig. 7 angegeben wurde. Es ist leicht begreiflich, dass beim Zurücksinken des Aryknorpels auch ein Wiederaufrichten durch die elastische Befestigung stattfinden muss. Indem die Achsen dieser Knorpel solcherweise den. früheren Stand (siehe Fig, 6) einnehmen, nähert sich der virtuelle Punkt $c$ wieder der Hinterfiäche des Ringes.

Zugleich mit dieser Bewegung findet aber eine andere statt. Denn wenn $a$ (Fig. 10) sich senkt, ist das auch der Fall mit $b$, der Processus muscularis, der durch die vorangehende Kontraktion des Posticus am Ringknorpel angedrückt gehalten wird. Dieser Processus liegt aber niedriger als $a$ und man muss solcherweise annehmen, dass er etwa auf gleicher Höhe wie dliegt mit Bezug auf die Trachealachse. Vielleicht liegt $b$ selbst niedriger. Das Verhältnis zwischen $d$ und $b$ wird also gedacht werden müssen, als dasjenige, welches zwischen $a^{\prime}$ und $b^{\prime}$, oder als das, welches zwischen $a^{\prime \prime}$ und $b^{\prime \prime}$ in $I I$ und $I I I$ von Fig. 5 gezeichnet ist.

Es leuchtet daher ein, dass beim Senken von $b$ in der Richtung der Trachealachse der Abstand' zwisehen $b$ und $a$ sich vergrössern muss. Da $d$ als fix zu gelten hat, so muss $b$ nach hinten rücken. Der Winkel $b c d$ wird gestreckt; $c$ muss sich um $a$ drehen und kommt in $k$ zu liegen, wenn $b$ bis in $l$ zurückgedreht ist. Wieder haben wir es mit der Kombination einer linearen Bewegung (Senkung) und einer 
Drehung zu tun. Auch hierbei findet eine Schraubenbewegung statt, jetzt aber der Richtung bei der Glottisschliessung entgegengesetzt.

Laryngoskopisch kann man die Überzeugung gewinnen, dass bei sehr tiefen Inspirationen die Eröffnung der Glottis so weit fortschreiten kann, dass die Stimmbänder ganz an die Wand des Larynx angedrückt erscheinen. Bei der gewöhnlichen Respiration wird aber diese Stellung bei weitem nicht erreicht. Ebensowenig findet man die Kadaverstellung, die einer tiefen Exspiration entspricht. Für gewöbnlich nehmen die Stimmbänder eine Mittelstellung zwischen diesen beiden extremen Zuständen ein. So fand Semon, dass bei ruhiger Respiration die Glottisweite durchschnittlich beim Manne $13,5 \mathrm{~mm}$, bei Frauen $11,5 \mathrm{~mm}$ betrug, während die Maasse für die Kadaverstellung 5 und $4 \mathrm{~mm}$ waren.

Die Mittelstellung der Glottis ist unabhängig von irgendwelcher Muskelwirkung des Kehlkopfes. Sie wird ganz und gar bestimmt durch das gegenseitige Verhältnis der beiden Kehlkopfhälften. Nur die Musculi sterno-thyreoidei und thyreo-hyoildei verhalten sich gewissermaassen aktiv als Folge der Hebung und Senkung des Brustbeines. Sie müssen also zu den Respirationsmuskeln gerechnet werden. Bei der gewöhnlichen Respiration wird ihre Aktivität sich wohl beschränken auf eine kleine Schwankung des Tonus.

Eben die Unabhängigkeit von Muskelwirkung macht die Glottisstellung sehr labil. Automatisch kann diese sich sofort den Luftdruckschwankungen anpassen, ohne dass diese mit der relativ trägen Kontraktion und Erschlaffung von Muskeln zu schaffen hat. Diese leichte, selbständige Anpassung ist von so überwiegender Bedeutung für den Körper, dass die auf Muskelwirkung beruhende Phonation nur sehr nebensächlich heissen kann.

Charakteristisch für den respiratorischen Kehlkopf ist seine Zweiteilung. Es ist nur zu natürlich, dass sich das auch in der Innervation kundgibt. Ein gemeinsamer Nerv für beide Hälften ist schwerlich denkbar ohne ein fortwährendes Zerren bei der funktionellen Verschiebung. Der Ringknorpel, der sich im Anschluss an den Brustinhalt bewegt, erfordert einen Nerv, der diese Bewegung ganz mitmachen kann. Der Recurrens markiert sozusagen diesen Zusammenhang mit den Brustorganen. Solcherweise lässt sich eine physiologische Basis ausfindig machen für sein merkwürdiges anatomisches Verhalten.

Vom nämlichen Gesichtspunkte aus scheint es uns auch begreiflich, dass die obere Kehlkopfhälfte absonderlich innerviert wird. Ihre relative Unabhängigkeit vindiziert einen eigenen Nerv.

E. Pflüger, Arehiv für Physiologie. Bd. 123. 
Wie man sieht, nehmen wir eine Scheidung im Innervationsgebiete an auf Grund der Verschiedenheit in Bewegungsrichtung bei der Funktion. Wir erwarten also, dass die Muskeln, die nur Beziehung haben auf die untere Hälfte des Larynx rom unteren Kehlkopfnerven, und dass die der oberen Hälfte vom Laryngeus superior versorgt werden. Darüber erhebt sich auch kein Zweifel. Nur mit Bezug auf Muskeln, die zwischen beiden Hälften ausgespannt sind, gibt es streitige Fragen. Am liebsten würde man an eine Innervation von beiden Seiten aus glauben. Aber dieser Schematismus trifft schon beim Musculus crico-thyreoïdeus nicht $\mathrm{zu}$, da dieser vom Laryngeus superior allein versorgt wird.

Viel umstritten ist die Frage der sensibelen Innervation des Kehlkopfes. Sie bleibt hier-ausserhalb unserer Betrachtungen, da wir die Glottis nur als Klappenapparat, d. h. von mechanischem Standpunkte aus, ins Auge fassen. Es gibt aber einen Punkt, der erwähnt zu werden verdient, nämlich die Reflexerregbarkeit.

Verschiedene Untersucher, unter denen Rosenthal !) genannt werden muss, haben Reizversuche am N. laryngeus superior angestellt. Sie fanden, dass zureichend starke elektrische Reizung der sensibeln Fasern dieses Nerven eine völlige Erschlaffung des Zwerchfells zur Folge hatte. Wir haben also einen Reflexbogen anzunehmen, der vom oberen Kehlkopf ausgehend, im Diaphragma einen Endpunkt findet. Hier aber erfolgt Erschlaffung, d. h. also eine exspiratorische Bewegung. Nach unserer Darstellung muss diese begleitet sein von einer Verengerung der Glottis. Die Abwehrbewegung des Larynx, denn so muss diese reflektorische Verkleinerung der Stimmritze wohl aufgefasst werden, kommt bier also in erster Linie auf dem Wege der Atmung zustande. Auch eine sich anschliessende phonetische Funktion des Kehlkopfes, wie z. B. der Husten, kann beschwerlich einen anderen Weg wählen. Auf zudringliche Weise scheint uns durch diesen Reflex der enge Zusammenhang von Glottis- und Atembewegungen betont zu werden.

Note: Meine abweichende Darstellung wird möglicherweise auf Widerspruch stossen. Ich bitte darum die Herren Opponenten, mir ihre Artikel zuzuschicken, da ich mich gerne, nach bestem Wissen, meiner Pflicht erledigen will.

1) Rosenthal, Die Atembewegungen und ihre Beziehungen zum Nervus vagus. Berlin 1862 . 